\title{
Feasibility and acceptability of methods to collect follow-up information from parents 12 months after their child's emergency admission to paediatric intensive care
}

Rachel Anne Pulham*1 BSc, Jo Wray*1 PhD, Yael Feinstein ${ }^{2}$ MD, Katherine Brown ${ }^{2}$ MD MPH, Christine Pierce ${ }^{3}$ FRCPCH, Simon Nadel ${ }^{4}$ MD, Nazima Pathan ${ }^{5}$ PhD, Elena Garralda ${ }^{6}$ FRCPsych FRCPCH, Padmanabhan Ramnarayan ${ }^{4,7}$ FRCPCH MD

1. Centre for Outcomes \& Experience Research in Children's Health, Illness \& Disability (ORCHID), Great Ormond Street Hospital for Children NHS Foundation Trust, London, United Kingdom

2. Cardiac Intensive Care Unit, Great Ormond Street Hospital for Children NHS Foundation Trust, London, United Kingdom

3. Paediatric Intensive Care Unit, Great Ormond Street Hospital for Children NHS Foundation Trust, London, United Kingdom

4. Paediatric Intensive Care Unit, St Mary's Hospital, Imperial College Healthcare NHS Trust, London, United Kingdom

5. Department of Paediatrics, University of Cambridge, United Kingdom

6. Centre for Psychiatry, Imperial College London, United Kingdom

7. Children's Acute Transport Service (CATS), Critical Care Division, Great Ormond Street Hospital for Children NHS Foundation Trust, London, United Kingdom

*Joint first author

\section{Corresponding author:}

Dr Padmanabhan Ramnarayan

Children's Acute Transport Service (CATS), Great Ormond Street Hospital

26-27 Boswell Street, London WC1N 3JZ

Telephone: +44 2074305850

Email: p.ramnarayan@gosh.nhs.uk

Copyright form disclosure: The authors have disclosed that they do not have any potential conflicts of interest. 


\begin{abstract}
Objective: To evaluate the feasibility and acceptability of different methods of collecting follow-up data from parents 12 months after their child's emergency admission to a paediatric intensive care unit (PICU).
\end{abstract}

Design: Mixed-methods explanatory sequential design.

Setting: One regional PICU transport service and three PICUs in England.

Patients: Children undergoing emergency transport to PICU recruited to an ongoing biomarker study whose parents consented to be contacted for follow-up 12 months after PICU admission.

Interventions: None

Measurements and Main Results: Parents/guardians who consented were asked to complete three questionnaires about their child's functional status, quality of life and behaviour 12 months after PICU admission. Parents were given a choice about method of questionnaire completion: postal, online or telephone interview, and also asked for telephone feedback about the process and the reasons for their choice. Of 486 parents who consented to be contacted at 12 months, 232 were successfully contacted. Consent to receive questionnaires was obtained in 218/232 (94\%). Of the 218 parents, 102 (47\%) chose to complete questionnaires online (with $77 \%$ completion rate); 91 (42\%) chose to complete postal questionnaires (48\% completion rate) and 25 (11\%) chose to complete questionnaires by telephone interview (44\% completion rate).

Conclusions: Parents expressed different preferences for follow up questionnaire completion. Response rates varied by completion method. Understanding and catering for 
parental preferences is an important factor in maximising response rates for follow-up studies in intensive care. 


\section{Introduction}

There is growing awareness that mortality has reduced in paediatric intensive care units

(PICUs) over recent years and there is increased morbidity in survivors.(1, 2) Consequently, the focus of health outcomes measurement is now shifting from mortality towards the longer term impact of intensive care admission.(3) We know that adverse physical, psychological or cognitive outcomes occur in $10-36 \%$ of children in studies of selected cohorts(4-8) and that children who are more severely ill at PICU admission and those admitted in an emergency appear to have worse outcomes at one and six months. $(9,10)$ Whilst there is clinical agreement regarding the importance of collecting data on outcomes and families advocate research priorities that focus on outcomes,(11) parental preferences regarding methods to collect routine follow up information in large cohorts is still unknown.(12)

Children requiring emergency admission to PICU, and specifically those who require emergency transport, have been poorly studied in terms of post-discharge outcomes. Since the centralisation of paediatric intensive care services in the UK, nearly 5000 children require emergency transport from their local hospital to a PICU each year.(13) Transported admissions account for half of all emergency PICU admissions; they require invasive interventions and experience long length of stay.(14) There is limited research on the longterm functioning of this cohort of children; most are discharged from PICU back to their local hospital or community services without any follow-up at the tertiary centre, in part due to the practical difficulties associated with following up a large number of children from geographically different locations. Outcome assessment and routine data collection are therefore challenging in these children. 
Face-to-face data collection with large cohorts of children from geographically diverse areas may be unfeasible, but use of questionnaires offers a method which can potentially be rolled out on a large scale, albeit with limitations. Evidence about methods of data collection suggests that adopting a mixed mode design for collecting questionnaire data caters for individuals' preferred methods of completion and therefore increases response rates(15-17) compared to participants who are not offered their preferred mode.(18) Social Exchange Theory proposes that if an individual perceives their costs to be minimized and benefits maximised, for example making the process as easy and convenient as possible, likeliness of response is increased.(16) Mode preference may also be influenced by demographic factors - specifically education, income, work status and age.(19, 20) A comprehensive evidence based strategy to improve response rates has been described, including (a) contacting participants before sending questionnaires, (b) follow-up contact and (c) providing non-responders with a second copy of the questionnaire.(21)

In order to address some of the gaps in current knowledge about long-term outcomes in children who require emergency transport to PICU our aims were to:

- Assess the feasibility and acceptability of using different methods of collecting follow-up data 12 months after emergency admission to PICU

- Determine whether response rates for different methods of data collection differed

- Determine the relative popularity of different methods of data collection.

\section{Methods}

This mixed-methods explanatory sequential follow-up study was nested within a larger 
prospective study (Biomarker of Acute Serious IIIness in Children, BASIC) which aimed to develop and validate biomarkers for distinguishing bacterial from viral infection and to predict death/severe disability at PICU discharge (NCT03238040). We chose to nest the followup study within BASIC (rather than as a standalone study) since detailed prospective clinical data collected during the PICU stay could be used to explore potential predictors of longer-term outcomes. The BASIC study methodology and cohort profile have been described in detail previously.(22) In brief, patients were recruited to BASIC if they were undergoing emergency transport to PICU by the Children's Acute Transport Service (CATS) for admission to one of three PICUs in England and had an indwelling arterial/venous and urinary catheter for sampling. Blood and urine samples were taken at first contact with the CATS team. Parents of children were first approached for consent to the BASIC study 24-48 hours after PICU admission (deferred consent model); closer to the time of PICU discharge, when the vital status of the child was clearer, research staff also asked parents for consent to be contacted for follow-up 12 months after the PICU admission. The project was granted ethical approval by the East Midlands-Nottingham 2 National Research Ethics Committee (13/EM/0399). Written informed consent was obtained from parents.

\section{Follow up questionnaires}

Twelve months after PICU admission, after establishing the child was alive via the NHS Summary of Care Record, parents were telephoned by a research assistant to ask if they would be willing to complete a general screening questionnaire and two validated questionnaires - the Pediatric Quality of Life Inventory(23) and the Child Behaviour Checklist(24) - about their child's functional status, quality of life and behaviour. Families were offered a choice of methods for completing the questionnaires: postal/paper 
questionnaire, online questionnaire (using REDCap survey functionality,(25) with a secure individual web link) or a telephone interview. The researcher organised a separate time with parents to call those who elected for telephone completion unless parents specifically suggested completing the questionnaire at the time of the initial call. Based on our initial pilot testing of the questionnaires with three families, we informed parents at the time of contact that the questionnaires would take approximately 20-30 minutes to complete.

Knowledge of existing evidence about effective follow up strategies from Social Exchange Theory(16) was used to maximise response rates: 1 . Participants were contacted prior to sending out questionnaires; 2 . After sending questionnaires out via parents' preferred mode (postal or online) a second copy or reminder was sent after two weeks; 3. A further questionnaire was sent at four weeks followed by telephone contact. All postal questionnaires were sent by first class post and participants were provided with a first class stamped-addressed envelope to return the completed questionnaires. Following questionnaire completion, by whichever mode, parents were telephoned to ask for feedback about the method of completion, reason for their choice, views of the process and any improvements, using a semi-structured interview format with probing if necessary. The researcher made contemporaneous notes during the call, documenting verbatim quotes where appropriate. Telephone interviews were continued until saturation was reached for each of the three methods of data collection.

\section{Analysis}

Descriptive statistics were used to characterise the sample and response rates were calculated for each stage of the recruitment process and for each mode of completion. We 
compared clinical characteristics of patients who were approached for consent for 12 month follow up with those not approached, those who consented for 12-month follow up with those who declined consent, and those who completed follow up questionnaires with those who did not. Statistical analysis was performed using Stata v14 (Stata Corp, College Station, Texas). The qualitative comments were thematically analysed, with two members of the research team independently coding the comments. Interview notes were read by each researcher and codes assigned to segments of data, with similar codes then merged to create themes. The researchers met to discuss the themes and to agree descriptive names assigned to them, with any differences being resolved by discussion until consensus was reached.

\section{Results}

In total, 674 children were recruited to the BASIC study over the 33-month study period (April 2014-Dec 2016). As shown in Figure 1, parents of 153 children were not approached for consent to be contacted at 12 months (died: 28; logistical reasons: 125). Logistical reasons included patients being admitted and discharged during a weekend or bank-holiday when there was no research nurse cover, parents being unavailable on multiple occasions and procedures happening with the child such that it was inappropriate to approach the parents at that specific time. Overall, 486/521 parents approached consented for 12 -month follow up contact (93\%) while 35 declined (7\%). As shown in Table 1, children approached were more likely to have respiratory and neurological diagnoses and less likely to have severe disability at baseline compared to those not approached; children who consented for 12-month contact were less likely to have a diagnosis of sepsis/infection compared to those who declined. Of 480 children eligible for 12 -month contact, no attempt was made to 
contact 161 families due to lack of research assistant support for the final 12 months of the study (34\%). Of the remainder, 232 (73\%) were successfully contacted at 12 months, of whom 218 consented to complete questionnaires, which represented $45 \%$ of those who had agreed at recruitment to be contacted but $68 \%$ of those whom we actually attempted to contact at 12 months. Completed questionnaires were received from 115 (53\%), with 9 (4\%) partially completing the questionnaires and $94(43 \%)$ not completing any questionnaires at all. Those who completed the questionnaires at 12 months $(n=124)$ were less likely to have co-morbid illnesses and spent a shorter duration in PICU compared to those who did not complete questionnaires $(n=94)$, as shown in Table 2 .

\section{Parental choice}

Of the 218 parents who consented to complete questionnaires, $102(47 \%)$ chose to receive the questionnaires online, 91 (42\%) chose to receive paper questionnaires and 25 (11\%) chose to complete questionnaires in a telephone interview.

\section{Completion rate}

Of the 102 parents who chose online completion, 70 (69\%) completed the questionnaire and there were a further $8(8 \%)$ parents who started but did not finish the online questionnaires, despite two reminders (Figure 2). For postal questionnaires, 43 of the 91 (47\%) who chose this method returned them, with one parent (1\%) only partially completing the questionnaire. Eleven parents completed the questionnaire by phone, representing $44 \%$ of those who chose this method.

Parental Feedback 
Fifty-four (25\%) families gave feedback on their involvement - 22 (41\%) online responders, 20 (37\%) postal responders and 12 (22\%) telephone responders. Two themes about parents' reasons for their choice of method were identified, related to convenience and ease of use.

\section{Convenience}

Parents discussed how their choice was influenced by what worked well with their daily routine, with time being a particular consideration:

"[Online] is most convenient to fit in with my life...I can do it at any time and don't have to remember to post it" (Online respondent)

One parent commented that online was better because they found it "really difficult to find the time to complete questionnaires" whilst another online respondent liked the "...flexibility of being able to do it when it was most convenient". Although the idea of being able to complete it when it suited was mentioned by both online and postal respondents, this was primarily identified by those who completed the questionnaires online rather than postally. However, several parents who chose postal completion talked about the possibility of missing something that was sent electronically:

"I chose to complete the questionnaire via post because I didn't want to miss it in my emails as I get so much junk that I can't be bothered to check them all" (postal respondent)

For those respondents who chose telephone completion, time was also an important consideration, with the flexibility of the researcher being key to the success of this method: "She [the researcher] called at a good time - it's just much easier for me. I'd just forget and I wanted to help" (Telephone respondent) 
Ease of use

Many of the parents described the reason for their choice as being related to the ease of use. Some parents simply described their choices as being 'the easiest' of all of the available options whilst others provided more detail. For example, one telephone respondent stated that it was "easier to talk than to write it [the responses]". Another felt telephone was the easiest because of other demands on her time:

"It is easier for me, it is difficult to find time because of small children. I would forget to post back or to check emails" (Telephone respondent)

For others it was more about the ease of completion in terms of the skills required:

"I can't read or write very well so telephone is best" (Telephone respondent)

One respondent described how postal completion gave her more time to finish it:

"It is easier to sit with time to think. Many online questionnaires time out during completion" (Postal respondent)

On-line respondents also identified their chosen method as being easy or the easiest for them. Some parents talked about an online questionnaire being simpler to access than either paper or phone and that that made it quicker and easier to complete. One parent described her particular situation at the time and how an online completion fitted in better:

"I am currently on a ward with my daughter so I have time where I don't have much to do.

Plus I wouldn't be at home to get the post" [Online respondent]

Fifty-two parents (96\%) would use the same method again whilst two (4\%) would prefer to complete online rather than by post. No respondents had any suggestions for improvements or alternative methods but some parents did comment on the fact that the questionnaire 
was straightforward to complete and how the attitude of the researcher was a positive factor in facilitating completion:

"I was approached by a very polite person who explained the reasons behind the work carefully. It was great." (Online respondent)

Parents also valued being given a choice about method of completion and being asked to provide feedback about their child, as illustrated in the following quote:

"I felt listened to and that my input was important, which is vital" (Online respondent)

Fifty-one (94\%) respondents said that they would be happy to complete future questionnaires for this research although some thought that the questionnaires were quite long and that not all questions were relevant for the youngest children.

\section{Discussion}

Collection of long-term outcome data is becomingly an increasingly important goal in paediatric intensive care, yet there is limited research about how best to collect this information routinely and in a cost-effective manner in a large cohort of patients from geographically different locations. Our findings show that it is feasible to collect follow up data 12 months after emergency transport and PICU admission in a reasonable proportion of children and that offering a choice of method for parents is potentially an important factor in maximising the response rate, allowing them to choose a method that suits their individual circumstances. By capturing reasons for parents' preferred method of completing questionnaires, we have also highlighted that parents chose options based on convenience, ease, speed and how easily they could incorporate them into their lives. 
Studies of long term outcome in PICU survivors have traditionally collected follow up data from parents or children either during a clinic visit (face-to-face) or through a postal or telephone questionnaire, with variable response rates.(9) The overall response rate in our study (57\% of those who consented at 12 months to complete the questionnaires) compared favourably with other studies, with the response rate for online completion being higher than previous studies. A recent study of long-term functional outcomes obtained a similar response rate to our study at 6 months and 3 years post-PICU discharge, but all data were collected by telephone.(26) Similarly, Knoester et al(27) reported a response rate of $57 \%$ in a study of quality of life 3 months after PICU discharge, using postal or face-to-face completion of questionnaires. In both studies the associated costs of collecting the data are likely to have been higher than in our study (due to researcher time to conduct the telephone or face to face interviews and time taken for data entry) but without a higher response rate. Prior evidence about methods of data collection supports the adoption of a mixed mode design for collecting questionnaire data. Catering for individuals' preferred methods of completion has been found to increase response rates in sociological studies(1517) compared to participants who are not offered their preferred mode(18). We based our methodology on a comprehensive evidence-based strategy(16) that has been previously suggested to improve response rates, including contacting participants before sending questionnaires, follow-up contact and providing non-responders with a second copy of the questionnaire.(21) Despite this approach, our response rate was not higher than some previously reported studies which have used more resource-intensive approaches such as face to face (including inviting families back to the treating hospital for a follow-up appointment) or telephone interviews. Although we clearly do not know how our response 
rate might have differed had we not adopted this strategy, it is likely, based on feedback we received from some families, that had we chosen one method for all families to use our overall response rate would have been lower. On the basis of this, we suggest that families should be offered a choice of mode for providing follow-up information, which we think will also increase the likelihood of enabling harder-to-reach families to complete questionnaires (e.g. those with poorer literacy levels may prefer to complete a telephone interview).

Our preliminary conclusions are that online completion is the most frequently preferred method for parents to submit follow up information, with the highest completion rate. In contrast to postal or telephone methods, partially completed online questionnaires could also be accessed by the research team, enabling some data to be obtained for analysis. Telephone administration was the least preferred method; it was also time-consuming for the research team and incurred hidden costs in terms of researcher time to administer the questionnaire. Although $42 \%$ of parents requested postal completion, this method had the lowest response rate and had costs associated with it in terms of printing and postage. Both telephone and postal completion also required additional resources for electronic data entry in contrast to direct online questionnaire completion.

Collection of follow-up data is clearly challenging but with the increase in the use of electronic resources it is becoming more feasible. Smartphone apps are increasingly being used to facilitate completion of e-questionnaires on a mobile device and this is an area which will continue to develop. $(28,29)$ At the time of undertaking this study we were not able to use text messaging or social media platforms as a means of contacting families but this is also something that could be explored further in future studies. Whilst face to face 
completion is still likely to be the most effective method, many children transported to PICU are not routinely followed up and, when they are, this is frequently not at the tertiary hospital. As collection of patient/parent reported outcome data becomes routine, we need to consider what information we collect at the first patient contact, about preferred methods of data collection and, importantly, what information patients and families are given about the reasons for collecting the data and what we do with it. Building on Social Exchange Theory, if patients and families are given information about the benefits to them, either directly or indirectly, of providing outcome data we suggest that it is more likely that they will return their questionnaires by whatever their chosen method is. Similarly, it is also important that we work with children and families to identify measures which they consider to be relevant, appropriate and acceptable to them to further maximise response rates.

Our study was limited by the fact that we did not collect information on parental demographics, education or work status. Future research should look at the role of these factors to determine whether there are other considerations to further tailor methods of data collection. We acknowledge that it would have been more informative if children were able to complete the questionnaires themselves, however this was not an option since the majority of children were $<5$ years. Non-completion and partial completion may have been influenced by the fact that some parents found the questionnaires time- and resourceheavy; parents of some of the youngest children also felt some of the questions were not relevant considering the age of their child, particularly those whose children were born prematurely and were therefore less than 12 months corrected age at the time of questionnaire completion. Furthermore, at the time of recruitment to BASIC we were unable to consent some parents to be contacted at 12 months for logistical reasons such as 
lack of availability of a research nurse during weekends and bank holidays and parents not being present in PICU, which may have introduced selection bias. For some of those who had agreed to be contacted we were also unable to contact them at 12 months to gain further consent for their participation and ascertain their preferred method of completion. Another potential bias is the likelihood that children with a diagnosis of sepsis or a higher level of disability on the POPC were not recruited either because they were not approached or they refused consent at a time when the child was still sick on the PICU. This is a challenging problem that needs to be addressed in future research, not least because it is important that the sickest patients are followed-up and their needs assessed as intuitively one might expect their ongoing requirement for additional resources to be greater than those whose functional status on admission to PICU is better. Interestingly, a year later and once outside the PICU setting, parents who provided follow-up data at 12 months had sicker children at PICU admission than those who did not provide follow-up data. One explanation is that these parents were more willing to be involved in the study because of how ill their children had been and their wish to 'give something back'. A further consideration is that a proportion of children recruited to BASIC did not have English as a first language and/or there were other cultural reasons why questionnaire completion was not feasible. Overall, the response rate was quite low and the sample size was relatively small. Future research should address why parents do not complete questionnaires in an attempt to improve response rates. Parents may, for example, not see the relevance of follow-up if their children are well or they may not see completion of a questionnaire as a priority if their child is receiving follow-up care and/or has complex needs. Understanding parents' reasons for non-completion would enable clinicians and researchers to tailor requests for follow-up information. 


\section{Conclusions}

Measurement of long-term outcomes in critical care survivors poses several challenges, not least of which is understanding how best to collect this information from large cohorts on a routine basis and when to approach families for consent for future contact. Understanding and facilitating parental preferences is an important consideration in maximising the response rate for follow-up studies. 
Figures

Figure 1: Flow diagram of study recruitment

Figure 2: Preferred methods of questionnaire completion and response rates 


\section{References}

1. Namachivayam P, Shann F, Shekerdemian L, et al. Three decades of pediatric intensive care: Who was admitted, what happened in intensive care, and what happened afterward. Pediatr Crit Care Med 2010;11(5):549-555.

2. Pollack MM, Holubkov R, Funai T, et al. Pediatric intensive care outcomes: development of new morbidities during pediatric critical care. Pediatr Crit Care Med 2014;15(9):821-827.

3. Typpo K, Mendelson J. After the Fairytale Ending: Functional Impairment After Pediatric Critical Illness. Pediatr Crit Care Med 2016;17(5):473-474.

4. $\quad$ Ong C, Lee JH, Leow MK, et al. Functional Outcomes and Physical Impairments in Pediatric Critical Care Survivors: A Scoping Review. Pediatric Critical Care Medicine 2016;17(5):e247-e259.

5. Rees G, Gledhill J, Garralda ME, et al. Psychiatric outcome following paediatric intensive care unit (PICU) admission: a cohort study. Intensive care medicine 2004;30(8):1607-1614.

6. Rennick JE, Johnston CC, Lambert SD, et al. Measuring psychological outcomes following pediatric intensive care unit hospitalization: Psychometric analysis of the Children's Critical Illness Impact Scale. Pediatric Critical Care Medicine 2011;12(6):635-642.

7. Gemke R, Bonsel GJ, Van Vught A. Long-term survival and state of health after paediatric intensive care. Archives of disease in childhood 1995;73(3):196-201.

8. Choong K, Al-Harbi S, Siu K, et al. Functional recovery following critical illness in children: the "wee-cover" pilot study. Pediatr Crit Care Med 2015;16(4):310-318.

9. Jones S, Rantell K, Stevens K, et al. Outcome at 6 months after admission for pediatric intensive care: a report of a national study of pediatric intensive care units in the United kingdom. Pediatrics 2006;118(5):2101-2108.

10. Ebrahim S, Singh S, Hutchison JS, et al. Adaptive behavior, functional outcomes, and quality of life outcomes of children requiring urgent ICU admission. Pediatric Critical Care Medicine 2013;14(1):10-18.

11. Manning JC, Hemingway P, Redsell SA. Survived so what? Identifying priorities for research with children and families post-paediatric intensive care unit. Nurs Crit Care 2018;23(2):68-74.

12. Namachivayam SP, Butt W. Outcomes After Pediatric Critical Illness: Important to Be Accurate. Pediatr Crit Care Med 2016;17(6):576-577.

13. Paediatric Intensive Care Audit Network (PICANet). 2014 Annual Report - Tables and Figures: Universities of Leeds and Leicester; 2014.

14. Ramnarayan P, Thiru K, Parslow RC, et al. Effect of specialist retrieval teams on outcomes in children admitted to paediatric intensive care units in England and Wales: a retrospective cohort study. Lancet 2010;376(9742):698-704.

15. Shih T-H, Fan X. Comparing response rates from web and mail surveys: A meta-analysis. Field methods 2008;20(3):249-271.

16. Dillman DA, Phelps $\mathrm{G}$, Tortora $\mathrm{R}$, et al. Response rate and measurement differences in mixed-mode surveys using mail, telephone, interactive voice response (IVR) and the Internet. Social Science Research 2009;38(1):1-18.

17. Millar MM, Dillman DA. Improving response to web and mixed-mode surveys. Public opinion quarterly 2011:nfr003.

18. Olson K, Smyth JD, Wood HM. Does giving people their preferred survey mode actually increase survey participation rates? An experimental examination. Public Opinion Quarterly 2012;76(4):611-635.

19. de Bernardo DH, Curtis A. Using Online and Paper Surveys The Effectiveness of Mixed-Mode Methodology for Populations Over 50. Research on Aging 2013;35(2):220-240.

20. Diment K, Garrett-Jones S. How demographic characteristics affect mode preference in a postal/web mixed-mode survey of Australian researchers. Social Science Computer Review 2007;25(3):410-417. 
21. Brealey SD, Atwell C, Bryan S, et al. Improving response rates using a monetary incentive for patient completion of questionnaires: an observational study. BMC medical research methodology 2007;7(1):1.

22. Feinstein $\mathrm{Y}$, Walker JC, Peters MJ, et al. Cohort profile of the Biomarkers of Acute Serious IIIness in Children (BASIC) study: a prospective multicentre cohort study in critically ill children. BMJ open 2018;8(11):e024729.

23. Varni JW, Seid M, Kurtin PS. PedsQL ${ }^{\text {TM }}$ 4.0: Reliability and validity of the Pediatric Quality of Life Inventory ${ }^{\mathrm{TM}}$ Version 4.0 Generic Core Scales in healthy and patient populations. Medical care 2001;39(8):800-812.

24. Achenbach TM. Achenbach system of empirically based assessment (ASEBA): Development, findings, theory, and applications: University of Vermont, Research Center of Children, Youth \& Families; 2009.

25. Harris PA, Taylor R, Thielke R, et al. Research electronic data capture (REDCap)--a metadatadriven methodology and workflow process for providing translational research informatics support. Journal of biomedical informatics 2009;42(2):377-381.

26. Pinto NP, Rhinesmith EW, Kim TY, et al. Long-term function after pediatric critical illness: results from the survivor outcomes study. Pediatric Critical Care Medicine 2017;18(3):e122-e130.

27. Knoester $\mathrm{H}$, Bronner MB, Bos AP, et al. Quality of life in children three and nine months after discharge from a paediatric intensive care unit: a prospective cohort study. Health and quality of life outcomes 2008;6(1):21.

28. Teixeira Neto NC, Lima YL, Almeida GPL, et al. Physiotherapy Questionnaires App to Deliver Main Musculoskeletal Assessment Questionnaires: Development and Validation Study. JMIR Rehabil Assist Technol 2018;5(1):e1.

29. Zhou L, Bao J, Parmanto B. Systematic Review Protocol to Assess the Effectiveness of Usability Questionnaires in mHealth App Studies. JMIR Res Protoc 2017;6(8):e151. 


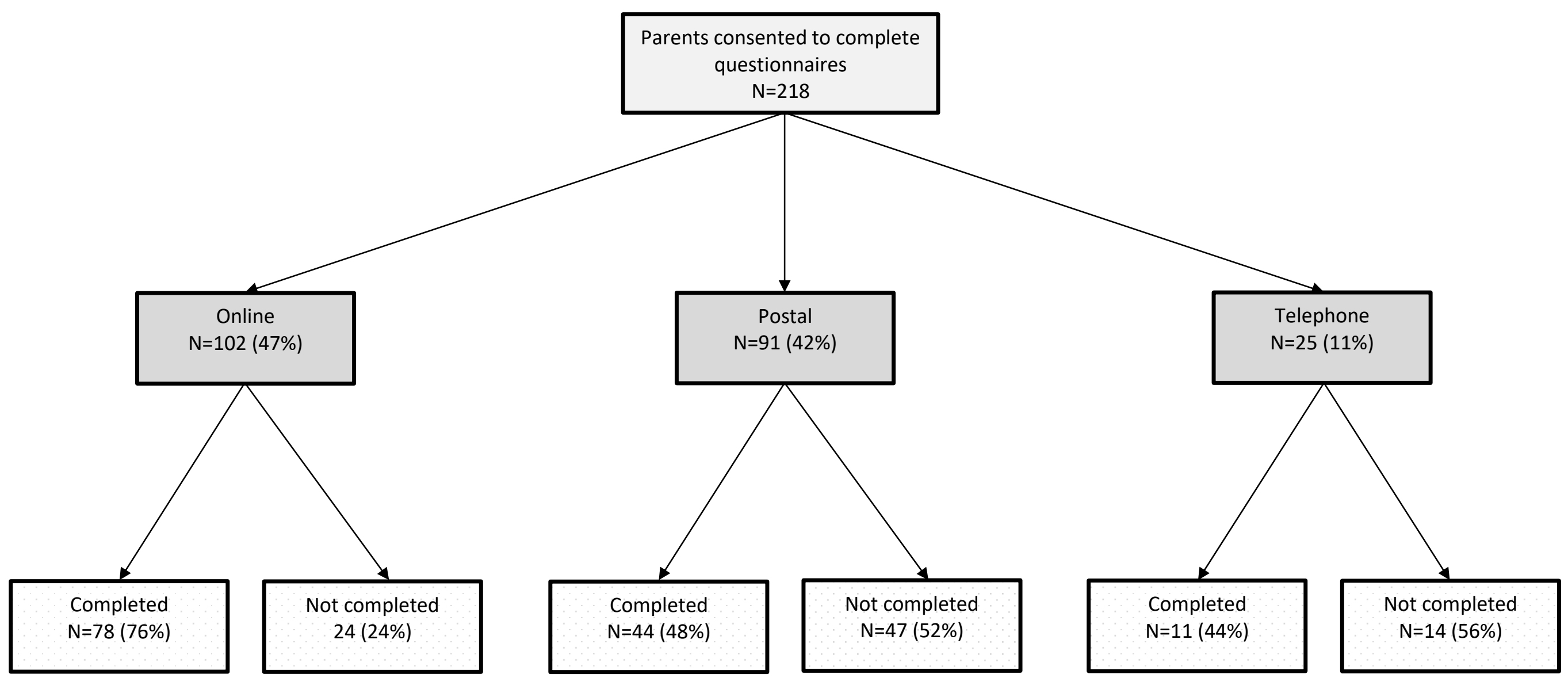

Figure 2: Preferred methods of questionnaire completion and response rates 